\title{
Les laboratoires d'analyses médicales et la métrologie
}

\author{
Didier Bozonnet \\ CGFL, 1 rue du Professeur Marion, 21000 Dijon France - Unicancer, 101 rue de Tolbiac 75654 Paris cedex 13 \\ France
}

\begin{abstract}
In France, biopathology laboratories should be accreditated according to ISO 15189. The metrology as a controlling of measurement process may confuse biologist or pathologist. Quality control is one aspect of the metrology process and rule the conformity of an analysis. This article focuses on 'preventive metrology' which provide confidence on what could be called the 'operational metrology'. Temperature is chosen as an example. The need of the fonctionnal process, defined before the analysis, is correlated to capability and uncertainty of the measurement instrument. The conformity of a measurement is also discussed towards to the reality of environnemental condition control.
\end{abstract}

\section{Les exigences de la norme ISO15189 [1]}

Si effectivement la métrologie nécessite l'acquisition de compétences et la mise à disposition de ressources, le laboratoire d'analyse médical ou d'anatomopathologie (LBM) a la possibilité de réaliser ses opérations de métrologie. Toutes ne sont pas «internalisables» et, pour certaines notamment les températures, cela apporte une réelle valeur ajoutée en termes de maîtrise du processus.

Le SH REF 02 [2] offre d'ailleurs cette possibilité dans son chapitre 5.3: «Le raccordement de ces équipements critiques de mesure (balances, pipettes, thermomètres, étalons,...) au Système international (SI) d'unité est assuré, selon le cas lorsqu'il est pertinent et possible :

.... soit par un étalonnage réalisé en interne par le LBM pour son propre compte; la preuve est alors le rapport sur les résultats (certificats d'étalonnage, constat de vérification, ...) émis par le LBM. Les aspects techniques sont évalués selon mes mêmes exigences que celles applicables aux laboratoires d'étalonnage selon la norme NF EN ISO/CEI 17025 [3]. »

Dans le cas d'un service de métrologie, qui appartient à un établissement de santé, le service métrologie doit ajouter aux exigences techniques de la norme ISO 17025, un système de management de la qualité (SMQ) fondé sur ce référentiel.

Si les SMQ du LBM et du service de métrologie sont différents, des accords de fonctionnement entre le LBM et le service métrologie assure la qualité de prestation requise. «Le service métrologie de l'organisme fait l'objet d'une évaluation spécifique par le COFRAC selon les mêmes règles en vigueur pour les laboratoires d'étalonnage. »

Un examen de biologie médical ou d'anatomopathologie (biopathologie) comporte trois processus : la phase pré analytique, analytique et postanalytique.

Dans la phase analytique, des courbes de calibrations utilisent des substances de références. Les contrôles qualité (contrôle interne ou CIQ et évaluation externe ou EEQ), permettent de statuer sur la performance d'un examen, notamment en évaluant et en suivant l'incertitude de la méthode, dans le cas des méthodes quantitatives. C'est, ce que je nommerai la métrologie opérationnelle qui en dernier ressort assure sa fiabilité Le détail de cette métrologie ne sera pas traité dans cet article mais je renvoie le lecteur vers les guides COFRAC SH GTA 14 [4] et SH GTA 06 [5]

La maîtrise «métrologique » en amont et en aval de l'analyse proprement dite permet la mise en œuvre de la phase analytique dans des conditions maîtrisées. Cela est d'autant plus important pour un examen de type qualitatif. On va retrouver ici, la conservation et la préparation des échantillons/ réactifs ...

C'est ce que j'appellerai la «métrologie préventive ».

Et c'est au moment du choix d'un nouvel examen, lors l'établissement du cahier des charges qu'il est nécessaire d'établir la criticité de tous les facteurs susceptibles d'influer sur la qualité finale de l'examen. 
L'outil de prédilection est l'analyse de risque. Alors, quant bien même cette gestion du risque soit une exigence de la norme ISO 15189 , elle est avant tout un outil qualité de maîtrise du processus d'analyse. Elle doit être conduite avec grande exhaustivité le plus en amont possible lors du choix d'un nouvel examen. Toutes les compétences doivent être représentées: biologiste/pathologiste, métrologue, fournisseur d'automate, informaticien, responsable qualité, ....

C'est d'ailleurs dans cette phase d'analyse du risque que doit émerger le besoin qui va permettre d'établir les exigences métrologiques des appareils de mesure des facteurs pouvant influer sur la qualité de l'examen (température, volume, vitesse, temps, ...)

\section{Comment formaliser le besoin ?}

En métrologie, il se traduit concrètement par l'établissement d'un intervalle de tolérance autour d'une valeur numérique d'une grandeur donnée. Dans l'absolu, tous les facteurs critiques devraient s'écrire sous la forme :

$$
X \pm a<\text { unité }>\text { ou } \leq X<\text { unité }>
$$

Cette valeur « a » représente l'Erreur Maximale Tolérée (EMT) du processus fonctionnel. Plus l'EMT est petit plus la grandeur associée est influente sur le résultat de l'analyse. A contrario, une EMT très large indique une grandeur dont l'influence est moindre sur l'échelle de gravité de l'analyse de risque.

Un processus fonctionnel peut être : une conservation à température maîtrisée, une vitesse de centrifugation, une opération de pipetage d'un réactif, la mesure d'un temps de réaction, le température d'activité enzymatique...

Et c'est à partir de la connaissance de l'EMT du processus fonctionnel que le processus de métrologie peut démarrer.

Dans une méthode de portée A, la bibliographie devrait permettre de trouver les valeurs d'EMT. Les notices des réactifs donnent aussi des informations. Mais nous verrons avec des exemples précis que ces dernières doivent être prises en considération prudemment, notamment sur les EMT de pipetage des réactifs et échantillons.

Dans une méthode de portée B, on établit ces EMT lors de l'étude de la robustesse de la méthode et l'outil de choix demeure le plan d'expérience. Non traitée dans cet article des références en libre accès internet permettent la mise en œuvre de cette méthodologie.

\section{Capabilité, incertitude et confirmation métrologique}

La capabilité d'un moyen de mesure définit l'aptitude d'un instrument à réaliser la mesure d'un paramètre qui se situe dans un intervalle de tolérance spécifié. [6]

La connaissance de la valeur de l'EMT fonctionnelle $\left(\mathrm{EMT}_{\text {fonc }}\right)$ permet de choisir un matériel de mesure CAPABLE.
Cette aptitude du matériel de mesure (minuteur, thermomètre, pipettes, tachymètre...) à mesurer la grandeur qui lui est attribuée (temps, température, volumes, vitesse angulaire, ...) est une valeur contractuelle définie par le LBM : la capabilité $\left(\mathrm{C}_{\mathrm{p}}\right)$

Son choix aura une incidence sur l'incertitude tolérée du processus de mesure.

Voyons deux cas. Dans le premier, la référence choisie pour réaliser la mesure est une référence primaire qui est envoyée directement à un prestataire dont l'étalon est raccordé aux étalons internationaux. Dans le jargon il est souvent dit que le prestataire est accrédité. Le LBM décide de sous-traiter ces opérations de métrologie. Nous verrons par la suite sur quels critères choisir un prestataire.

Le facteur de capabilité est généralement fixé à 4. C'est ce que dans le jargon le biologiste appelle la « règle du quart $»$.

Concrètement une capabilité de 4 signifie que pour mesurer un processus fonctionnel, le métrologue choisit un matériel ou plutôt une méthode de mesurage 4 fois plus capable.

Dans le second cas, le LBM choisit de réaliser lui-même des étalonnages, des sondes de température par exemple.

Il utilise alors des références secondaires qu'il étalonne lui-même par rapport à un étalon primaire. Le raccordement aux unités internationales est assuré par l'envoi de l'étalon primaire à un prestataire accrédité.

La chaîne de mesure en termes de capabilité peut être choisie comme suit [7] :

$\mathrm{Cp}=2$ entre processus fonctionnel et étalon secondaire $\mathrm{Cp}=4$ entre étalon secondaire et étalon primaire

$\mathrm{Si}$ le processus de mesure est pris dans sa globalité, l'étalon primaire est 8 fois plus capable que le processus fonctionnel dans lequel il est engagé.

La relation suivante relie la capabilité du processus de mesure $\left(C_{p}\right)$, l'EMT $_{\text {fonc }} \mathrm{du}$ processus fonctionnel et l'incertitude recherchée du processus de mesure (Ur) :

$$
C_{p}=\frac{E M T_{\text {fonc }}}{U_{r}}
$$

Connaissant l'EMT $\mathrm{l}_{\text {fonc }}$ et $\mathrm{C}_{\mathrm{p}}$, il est déduit que l'incertitude du processus de mesure (Ur) ne devra pas dépasser la valeur :

$$
\frac{E M T_{\text {fonc }}}{C_{p}}
$$

C'est la confirmation métrologique qui permet de conclure si l'étalonnage répond aux exigences du processus fonctionnel.

Exemple :

La conservation d'un réactif est entre +2 et $+8^{\circ} \mathrm{C}$, soit une $\mathrm{EMT}_{\text {fonc }}$ de $3^{\circ} \mathrm{C}$.

Le LBM choisit d'utiliser des sondes de températures secondaires mini-maxi pour effectuer la surveillance de l'enceinte thermostatique raccordées à une sonde primaire qui est envoyée à un prestataire accrédité. 
Il fixe un $\mathrm{Cp}$ de 2 entre sonde secondaire et $\mathrm{EMT}_{\text {fonc }}$ et un $\mathrm{C}_{\mathrm{p}}$ de 4 entre sonde secondaire et sonde primaire.

La confirmation métrologique de la sonde secondaire sera confirmée si l'incertitude de la sonde secondaire n'excède pas $1.5^{\circ} \mathrm{C}$. La confirmation métrologique de la sonde primaire sera confirmée si l'incertitude de la sonde primaire n'excède pas $0.38^{\circ} \mathrm{C}$ (incertitude recherchée).

La confirmation métrologique lors de l'exploitation d'un certificat d'étalonnage consistera donc à comparer la valeur d'incertitude du certificat avec l'incertitude recherchée. Cette confirmation métrologique est à réaliser aussi bien pour un étalon primaire que pour un étalon secondaire.

\section{Evaluer les incertitudes}

Une incertitude ne peut être rigoureusement déterminée s'il la méthode de mesurage n'est pas formellement établie. Cette première étape obligatoire se schématise bien sur un logigramme.

Ensuite avec l'outil qualité des 5M (Méthode, Main d'œuvre, Milieu, Moyen, Matériel) [8], le budget d'incertitude est établi. Même si certains facteurs d'incertitude seront considérés négligeables lors de la phase de quantification, il faut être exhaustif à ce stade.

Le GUM [9] détaille et démontre la quantification des incertitudes.

Pour résumer il y a deux types d'incertitude : type A et type B. Une incertitude de type A peut être quantifiée si on réalise une série de mesure en condition de répétabilité. L'incertitude-type $\left(\mathrm{u}_{\mathrm{c}}\right)$ d'une série de ' $\mathrm{n}$ ' mesure, quantifiée en calculant l'écart-type expérimental (s), est calculée comme suit:

$$
u_{c}=b_{n} \frac{s}{\sqrt{n}}
$$

$b_{n}$ est un facteur correctif qui tient compte du nombre de mesure réalisée. En effet plus le nombre de mesure est limité, plus l'écart-type expérimental est biaisé. [10], [11]

Dans une incertitude de type A il est supposé que les ' $n$ ' mesures de l'ensemble de la population statistique suivent une loi normale ou gaussienne.

La surface sous la courbe est l'ensemble des valeurs possibles pour une grandeur. $95 \%$ des valeurs représente 2 écart-type.

Sur un certificat d'étalonnage la valeur d'incertitude indiquée (U) est une incertitude de type A; c'est l'incertitude élargie. L'incertitude-type $\left(\mathrm{u}_{\mathrm{c}}\right)$ associée est égale à :

$$
u_{c}=U / 2
$$

La valeur 2 correspond à celle qui est mentionnée sur le certificat comme le facteur d'élargissement (2 écarttype).

Dans les cas où il n'y a pas d'indication claire de quantification de l'incertitude, il faut postuler une loi a priori. On parle d'incertitude de type B.
Avec la loi normale (4) deux autres types de lois sont attribuables aux facteurs d'incertitude pour les grandeurs rencontrés en laboratoire de biopathologie :

La loi rectangle avec :

ou

$$
\begin{gathered}
u_{c i}=\text { étendue } / 2 \sqrt{3} \\
u_{c i}=\text { demi- étendue } / \sqrt{3}
\end{gathered}
$$

On applique cette loi pour quantifier des incertitudestype concernant: la résolution, la dérive d'un instrument, la spécification d'un fournisseur, ....

La loi triangle avec

$$
u_{c i}=\text { demi }- \text { étendue } / \sqrt{6}
$$

On applique cette loi pour quantifier l'incertitude-type de l'influence de la pipette ou de la fiole pour laquelle le fournisseur annonce par exemple $100 \mathrm{~mL} \pm 0.1 \mathrm{~mL}$ à $20^{\circ} \mathrm{C}$.

Attention l'influence de l'opération du pipetage sur la mesure est un autre facteur d'incertitude.

D'autres lois existent et des nombreuses références sont disponibles.

Une fois les facteurs d'incertitude influents quantifiés en incertitude-type, l'incertitude-type globale du processus de mesure $\left(\mathrm{u}_{\mathrm{c}}\right)$ est estimé par la relation :

$$
u_{c}=\sqrt{\sum\left(u_{c i}^{2}\right)}
$$

Par la formule (4) est estimée l'incertitude élargie (U) du processus de mesure.

\section{Performance optimale d'un appareil de mesure?}

Jusqu'ici il a été permis de choisir un appareil de mesure pour une situation donnée, par exemple surveiller une enceinte thermostatique ayant une $\mathrm{EMT}_{\text {fonc }}$ de $\pm 3^{\circ} \mathrm{C}$.

Maintenant on souhaite utiliser le même type d'appareil et le même processus de mesurage de la sonde pour surveiller une étuve avec une $\mathrm{EMT}_{\text {fonc }}$ de $\pm 1^{\circ} \mathrm{C}$. L'appareil de mesure sera-t-il capable de réaliser cette surveillance ?

Le point de départ est de rechercher une donnée technique qui figure dans le catalogue d'achat; c'est la valeur de "précision » (anglicisme à ne pas employer en métrologie) ou justesse ou erreur systématique. C'est son $\mathrm{EMT}_{\text {app. }}$

Cette $\mathrm{EMT}_{\text {app }}$ peut être traduit en incertitude-type avec la formule (6).

En fixant une capabilité de 2 pour le moyen de mesurage, nous allons pouvoir déterminer l'EMT equ minimale de l'équipement fonctionnel qui pourra être utilisé par la relation :

$$
E M T_{\text {fonc }}=\frac{4 E M T_{\text {apm }}}{\sqrt{3}}
$$

Cette formule renvoie une valeur informative. Effectivement tout dépend de la fiabilité de la valeur de "précision » fournie par le constructeur de l'appareil 
de mesure. Elle va orienter le métrologue sur la méthode de mesurage la plus en adéquation avec son parc d'enceintes.

\section{Démonstration :}

L'incertitude type associée à la valeur de 'précision' du constructeur de l'appareil de mesure est :

$$
u_{c}=\frac{E M T_{a p m}}{\sqrt{3}}
$$

La formule (9) est démontrée en combinant (10) et les formules suivantes :

Exemple :

$$
\begin{gathered}
C_{p}=\frac{E M T_{\text {fonc }}}{U_{r}} \\
U_{r}=2 u_{c}
\end{gathered}
$$

Précision constructeur d'une sonde de température : \pm $0,5^{\circ} \mathrm{C}$

Méthode de mesurage: utilisation d'une sonde secondaire mini maxi avec $\mathrm{C}_{\mathrm{p}}$ de 2 raccordée à une sonde primaire $\left(\mathrm{C}_{\mathrm{p}}=4\right)$

Dans ces conditions l'EMT minimale de l'appareil fonctionnel devra être supérieur à $1.2^{\circ} \mathrm{C}$.

En conséquence, le processus de mesurage, qui donne satisfaction pour une enceinte ayant une EMT de $3^{\circ} \mathrm{C}$ ne pourra pas être utilisé pour une étuve ayant une EMT de $1,0^{\circ} \mathrm{C}$ qu'avec prudence.

\section{Quelle alternative alors?}

Premier cas: la méthode d'étalonnage de la sonde secondaire est conservée car des étalonnages successifs montrent que l'incertitude d'étalonnage est en deçà de $0.5^{\circ} \mathrm{C}$ en appliquant la formule (2).

Second cas : la sonde secondaire n'est pas étalonnée en interne mais envoyée à un prestataire accrédité. Cela aura pour conséquence de diminuer de fait l'incertitude du à l'opération d'étalonnage secondaire.

Troisième cas : une sonde plus performante est choisie («précision» plus faible) avec envoi ou non à un prestataire accrédité pour étalonnage.

\section{Incertitude et zone de conformité}

L'incertitude du moyen de mesure encadre les valeurs de spécification du moyen fonctionnel [12].

La zone ainsi définie autour de la spécification est une zone de doute, d'incertitude.

Statistiquement, la zone d'incertitude élargie contient $5 \%$ de valeurs qui peuvent être déclarées non-conforme alors qu'elles sont conformes.

Si effectivement, plusieurs mesures consécutives dans un relevé journalier sont constatées dans cette zone, alors une alerte doit être générée.

Pour autant la présence d'une valeur aléatoire ne présente pas de risque majeur pour l'examen et peut être déclarée conforme. Et c'est dans ce genre de situation que le bon sens du métrologue associée à l'expertise du biologiste/pathologiste doit se manifester.

Tout d'abord rappelons que la métrologie, d'une enceinte par exemple, est de la métrologie préventive et que c'est la métrologie opérationnelle avec le suivi des contrôles de qualité et des incertitudes associées qui assure in fine la maîtrise du processus analytique (suivi des contrôles qualité).
D'autre part, pour certains réactifs il n'y pas de de chaine du froid à proprement parler; les réactifs sont amenés à température ambiante, analysés puis remis dans l'enceinte froide de stockage.

Et finalement certains fournisseurs mettent à disposition des fiches de stress de réactifs et contrôles. Pour certaines d'entre elles le stress du transport est pris en compte mais les conditions du transport ne sont pas maîtrisés. Prenons par exemple le transport des réactifs d'hémostase qui peuvent subir, selon la fiche de stress, durant $24 \mathrm{~h}$ un stress entre $0^{\circ} \mathrm{C}$ et $30^{\circ} \mathrm{C}$. Ils sont expédiés dans un container polystyrène qui ne contient aucun pack froid.

Des essais de transport d'échantillons biologiques réalisés dans de telles conditions montrent que dans un véhicule où la température n'est pas maîtrisée les conditions climatiques en France ne permettent guère de garantir cet intervalle $0-30^{\circ} \mathrm{C}$. Ces fiches de stress ne sont pas vraiment représentatives des conditions réelles de transport. Alors, même si l'examen est en portée A avec suivi des recommandations fournisseurs, une valeur dans la zone d'incertitude engendre un risque quasi nulle pour la qualité de l'examen.

\section{Perspectives}

De nombreux laboratoires de biopathologie décident d'externaliser la métrologie des équipements pour la simple raison de ne pas maîtriser les compétences associées. Mais, même externalisé, le processus de métrologie ne consiste pas simplement à recevoir un certificat d'étalonnage ou de vérification et y apposer son paraphe. C'était l'objectif de cet article, vous orienter vers l'acquisition des compétences nécessaires pour avoir une symbiose entre besoin / exigences métrologiques et prise de décision. D'ailleurs le bon sens devrait aussi être une compétence en soi.

\section{Références}

[1] Norme NF/EN ISO 15189 : Laboratoires d'analyses de biologie médicale - exigences concernant la qualité et la compétence.

[2] Document Cofrac SH REF 02 : Recueil des exigences spécifiques pour l'accréditation des laboratoires de biologie médicale selon la norme NF EN ISO 15189 : 2012.

[3] Norme NF EN ISO/CEI 17025 : Exigences générales concernant la compétence des laboratoires d'étalonnage et d'essais.

[4] Document Cofrac SH GTA 14: guide technique d'accréditation pour l'évaluation des incertitudes de mesure en biologie médicales.

[5] Document Cofrac SH GTA 06: guide technique d'accréditation: contrôle de qualité en biologie médicale.

[6] JM Pou, Contrôle Essais Mesures n ${ }^{\circ} 39$ (mai 2012)

[7] Commission SFSTP, D. Louvel, C. Barbier, MD Blanchin, MC Bonenfant, .C Chmieliewsky, X. Dua, R. Dybiak, C. Imbernon, C.Lebranchu, L. Louvet, M 
Vandenhende, STP Pharma pratiques, 20, 1, (2010)

[8] : formation e-learning du lne disponible sur le site https://e-formation.lne.fr/

[9] : GUM JCGM 2008 disponible sur le site http://www.bipm.org/fr/publications/guides/ [10]: L'article «Ecart-type expérimental : quelle formule choisir? » disponible sur la page http:/www.deltamu.fr,

[11] : Engineering Statistics Handbook au §6.3.2 disponible sur Internet : http:/www.itl.nist.gov/div898/handbook/

[12] JM Pou, Contrôle Essais Mesures $n^{\circ} 11$ (avril 2005) pp 55-58 